\title{
From bench to clinic
}

\author{
Ashok Agarwal ${ }^{1}$, Chak-Lam $\mathrm{Cho}^{2}$, Ahmad Majzoub ${ }^{3}$, Sandro C. Esteves ${ }^{4}$ \\ ${ }^{1}$ American Center for Reproductive Medicine, Cleveland Clinic, Cleveland, OH, USA; ${ }^{2}$ Division of Urology, Department of Surgery, Kwong \\ Wah Hospital, Hong Kong, China; ${ }^{3}$ Department of Urology, Hamad Medical Corporation, Doha, Qatar; ${ }^{4}$ ANDROFERT, Andrology and Human \\ Reproduction Clinic, Referral Center for Male Reproduction, Campinas, SP, Brazil \\ Correspondence to: Ashok Agarwal. Professor and Director, American Center for Reproductive Medicine, Cleveland Clinic, Mail Code X-11, 10681 \\ Carnegie Avenue, Cleveland, OH 44195, USA. Email: AGARWAA@ccf.org. \\ Response to: Ward WS. Eight tests for sperm DNA fragmentation and their roles in the clinic. Transl Androl Urol 2017;6:S468-70.
}

Submitted Apr 13, 2017. Accepted for publication Apr 13, 2017.

doi: $10.21037 /$ tau.2017.04.23

View this article at: http://dx.doi.org/10.21037/tau.2017.04.23

Dr. Ward in his commentary summarized the currently available sperm DNA fragmentation (SDF) tests. He classified the eight SDF assays into four groups based on the different target sites and illustrated the grouping in a diagram to simplify understanding of the readers (1). We would like to supplement, in the following sessions, by (I) further solidifying the current clinical evidence of SDF, and (II) discussing means to move SDF from laboratories to clinics.

It is uncertain if one assay is superior over another since the exact nature of DNA fragmentation detected and their implications are not fully understood. In fact, the different type of SDF detected by each assay may be complementary to each other in different clinical settings which warrant further research. Even in absence of a more complete understanding about the nature of SDF, these tests have shown its promise in the workup of infertile men. There is a clear association between high SDF and decreased pregnancy rates in natural conception and intrauterine insemination (IUI) (2). Emerging evidence also suggests a negative impact of high SDF on pregnancy outcomes in in vitro fertilization (IVF) and intracytoplasmic sperm injection (ICSI) cycles (2). Sperm Chromatin Structure Assay (SCSA) is the first assay introduced clinically in the detection of damaged sperm DNA and altered proteins in sperm nuclei via flow cytometry of acridine orange stained sperm (3). The test results are stable over time in healthy individual (4). The correlation between sperm DNA integrity measured by SCSA and natural pregnancy outcome has been demonstrated in the Danish first pregnancy planners study. The study showed that the time-to-pregnancy increased with sperm DNA fragmentation index (DFI) in men who became infertile with DFI exceeding 30\% (5). A meta-analysis involving 616 couples showed that high DFI measured by SCSA was associated with a failure to achieve natural pregnancy with an odds ratio of 7.01 (6). The currently established clinical threshold of DFI is $25 \%$ for placing men into a statistical probability of longer time to natural pregnancy, low odds of IUI pregnancy, more miscarriages or no pregnancy (7). Furthermore, TUNEL assay has been shown to have high correlation with SCSA $(r=0.87)$ by using the same flow cytometer in the same laboratory (8). The correlation between TUNEL result and assisted reproductive technique (ART) outcomes has also been reported. SDF measured by TUNEL was shown to predict IUI outcomes and no pregnancy was achieved with $>12 \%$ SDF in the sample (9). The precision and reproducibility of TUNEL assay in a recent study showed that a high correlation in result $(\mathrm{r}=0.94)$ could be achieved between laboratories by using standardized protocol (10). SDF assays will firmly establish its role in the management of infertile couples with further clinical data.

To enhance the utilization of SDF as a new test of sperm function requires that, in addition to the available laboratory and clinical data, effort should be spent in popularizing the test among clinicians. First of all, deficiency in the current practice and a need for new advancement must be recognized. The limitation of semen analysis as the cornerstone of the male fertility assessment tool should be noticed as there is a significant overlap in semen parameters between fertile and infertile men (11). In fact, semen analysis failed to detect any abnormality in up to $30 \%$ of infertile men based on the current workup algorithm (12). Despite all the 
advances in ART, the use of ICSI as the treatment of male infertility resulted in a live birth of no more than $30 \%$ (13). Therefore, the potential of SDF test in providing additional information for male infertility patients should be conveyed to the clinicians. The practice recommendations by Agarwal et al. (14) attempted to bridge the gap between laboratory and clinic by suggesting wider clinical application of SDF tests in specific clinical scenarios based on the best current evidence. We believe it is an important first step to move the test from bench to clinic and benefit the infertile couples. Furthermore, the cost of SDF testing will probably be justified in face of the high cost of ART. A more targeted selection of ART technique based on SDF testing result will be a more economical choice than the trial-and-error approach of repeated ART cycles. In one study, Esteves et al. evaluated the use of testicular in preference over ejaculated sperm for ICSI in men with high SDF in semen. The authors showed that the number needed to treat by testicular compared to ejaculated sperm to obtain an additional live birth per fresh transfer cycles was 4.9 (95\% CI: 2.8-16.8) (15). Added to this, the risk of ART cycles and multiple gestations on both female partner and fetus can be reduced with appropriate selection of ART which will offer the highest success rate. We argue that the added information provided by the non-invasive SDF tests should lead to a better counselling of infertile couples in the busy clinics. Familiarity of the SDF tests amongst the clinicians is the missing link. Confidence in incorporating the test result in treatment decisions will certainly increase with time and more extensive use of the test.

\section{Acknowledgements}

None.

\section{Footnote}

Conflicts of Interest: The authors have no conflicts of interest to declare.

\section{References}

1. Ward WS. Eight tests for sperm DNA fragmentation and their roles in the clinic. Transl Androl Urol 2017;6:S468-70.

2. Agarwal A, Cho CL, Esteves SC. Should we evaluate and treat sperm DNA fragmentation? Curr Opin Obstet Gynecol 2016;28:164-71.

3. Evenson DP. The Sperm Chromatin Structure Assay
$\left(\mathrm{SCSA}\left({ }^{\circledR}\right)\right)$ and other sperm DNA fragmentation tests for evaluation of sperm nuclear DNA integrity as related to fertility. Anim Reprod Sci 2016;169:56-75.

4. Spanò M, Kolstad AH, Larsen SB, et al. The applicability of the flow cytometric sperm chromatin structure assay in epidemiological studies. Asclepios. Hum Reprod 1998;13:2495-505.

5. Spanò M, Bonde JP, Hjøllund HI, et al. Sperm chromatin damage impairs human fertility. The Danish First Pregnancy Planner Study Team. Fertil Steril 2000;73:43-50.

6. Zini A. Are sperm chromatin and DNA defects relevant in the clinic? Syst Biol Reprod Med 2011;57:78-85.

7. Evenson DP. Sperm chromatin structure assay (SCSA®). Methods Mol Biol 2013;927:147-64.

8. Gorczyca W, Traganos F, Jesionowska H, et al. Presence of DNA strand breaks and increased sensitivity of DNA in situ to denaturation in abnormal human sperm cells: analogy to apoptosis of somatic cells. Exp Cell Res 1993;207:202-5.

9. Duran EH, Morshedi M, Taylor S, et al. Sperm DNA quality predicts intrauterine insemination outcome: a prospective cohort study. Hum Reprod 2002;17:3122-8.

10. Ribeiro S, Sharma R, Gupta S, et al. Inter- and intralaboratory standardization of TUNEL assay for assessment of sperm DNA fragmentation. Andrology 2017;5:477-85.

11. Guzick DS, Overstreet JW, Factor-Litvak P, et al. Sperm morphology, motility, and concentration in fertile and infertile men. N Engl J Med 2001;345:1388-93.

12. Isaksson R, Tiitinen A. Present concept of unexplained infertility. Gynecol Endocrinol 2004;18:278-90.

13. Neri QV, Tanaka N, Wang A, et al. Intracytoplasmic sperm injection. Accomplishments and qualms. Minerva Ginecol 2004;56:189-96.

14. Agarwal A, Majzoub A, Esteves SC, et al. Clinical utility of sperm DNA fragmentation testing: practice recommendations based on clinical scenarios. Transl Androl Urol 2016;5:935-50.

15. Esteves SC, Sánchez-Martín F, Sánchez-Martín P, et al. Comparison of reproductive outcome in oligozoospermic men with high sperm DNA fragmentation undergoing intracytoplasmic sperm injection with ejaculated and testicular sperm. Fertil Steril 2015;104:1398-405.

Cite this article as: Agarwal A, Cho CL, Majzoub A, Esteves SC. From bench to clinic. Transl Androl Urol 2017;6(Suppl 4):S471-S472. doi: 10.21037/tau.2017.04.23 